\title{
A case report of tongue metastasis from lung squamous cell carcinoma and literature review: Erratum
}

In the article, "A case report of tongue metastasis from lung squamous cell carcinoma and literature review", ${ }^{[1]}$ which appeared in Volume 96, Issue 40 of Medicine, Dr. Yi-ping Han appeared incorrectly as Yipin Han.

\section{Reference}

[1] Cheng X, Hu Z, Han Y. A case report of tongue metastasis from lung squamous cell carcinoma and literature review. Medicine. 96;40:e8208. 